\title{
On Multi-Dimensional and Noisy Quadratic Signaling Games and Affine Equilibria
}

\author{
Serkan Sarıtaş, Serdar Yüksel, and Sinan Gezici
}

\begin{abstract}
This study investigates extensions of the quadratic cheap talk and signaling game problem, which has been introduced in the economics literature. Two main contributions of this study are the extension of Crawford and Sobel's cheap talk formulation to multi-dimensional sources, and the extension to noisy channel setups as a signaling game problem. We show that, in the presence of misalignment, the quantized nature of all equilibrium policies holds for any scalar random source. It is shown that for multi-dimensional setups, unlike the scalar case, equilibrium policies may be of non-quantized nature, and even linear. In the noisy setup, a Gaussian source is to be transmitted over an additive Gaussian channel. The goals of the encoder and the decoder are misaligned by a bias term and encoder's cost also includes a power term scaled by a multiplier. Conditions for the existence of affine equilibrium policies as well as general informative equilibria are presented for both the scalar and multi-dimensional setups.
\end{abstract}

\section{INTRODUCTION}

Signaling games and cheap talk are concerned with a class of Bayesian games where an informed decision maker transmits information to another decision maker and the information transmission policy can be viewed as the action of this decision maker. Signaling game and its specific case cheap talk can be viewed in the classical communications theory setup with the encoder as the sender and the decoder as the receiver taking part in a team decision problem where the encoder and the decoder have aligned objectives. Namely, the sender and receiver reach the most informative equilibrium. In this case, the fields of information theory and estimation theory have studied such problems extensively.

Such problems find applications in network control systems when a communication channel is present among competitive and non-cooperative decision makers [1]. One may consider a utility company which wishes to inform users regarding pricing information; if the utility company and the users engage in selfish behaviour, it may be beneficial for the utility company to hide information and the users to be strategic about how they interpret the given information. Another application area is smart-grids; there may be corrupted sensors with misaligned goals in the system [2] and

S. Sarıtas and S. Gezici are with the Department of Electrical and Electronics Engineering, Bilkent University, 06800, Ankara, Turkey. Emails: \{serkan,gezici\}@ee.bilkent.edu.tr. S. Yüksel is with the Department of Mathematics and Statistics, Queen's University, Kingston, Ontario, Canada, K7L 3N6. Email: yuksel@mast.queensu.ca.

This research was supported in part by the Natural Sciences and Engineering Research Council (NSERC) of Canada, The Scientific and Technological Research Council of Turkey (TÜBITAK) and the Distinguished Young Scientist Award of Turkish Academy of Sciences (TUBAGEBIP 2013). the security of the smart-grid infrastructure can be analyzed by applying the game theoretic approach to the interaction between an attacker and a defender [3], [4]. One further area of application is recommender systems (as in rating agencies) [5].

This study investigates extensions of the quadratic cheap talk and signaling game problem studied by Crawford and Sobel [6] in the economics literature. In this literature; there have been a number of related contributions, which we briefly review in the following: In [7], Krishna and Morgan study the setup in [6] with two senders; in [8], Shintaro studies an unbounded state space setup with two senders. Moreover, multi-dimensional cheap talk with single and multiple senders is analyzed in [9]-[12] under various considerations. Andreas et.al. [13] add uniform noise between the sender and receiver. Furthermore, there are some contributions which modify the information structure in Crawford and Sobel's game setup. In [14], the sender knows that the receiver has partial information about his/her private information; whereas the sender doesn't know this in [15], [16]. Golosov et al. [17] study Crawford and Sobel's game setup in a finite horizon environment where, in each period, a privately informed sender sends a message and a receiver takes an action.

In the control community, recently, there have been few studies: [18] considered a Gaussian cheap talk game with quadratic cost functions where the analysis considers Stackelberg equilibria, for a class of single- and multi-terminal setups and where linear equilibria have been studied. For the setup of Crawford and Sobel but when the source admits an exponentially distributed real random variable, [19] establishes the discrete-nature of equilibria, and obtains the equilibrium bins with finite upper bounds on the number of bins under any equilibrium in addition to some structural results on informative equilibria for general sources.

\section{A. Contributions of this Paper}

The main contributions of this study are the extension of Crawford and Sobel's cheap talk formulation to multidimensional sources, and the extension to noisy channel setups (as a signaling game) where a Gaussian source and a Gaussian channel are assumed. We show that for multidimensional setups, unlike the scalar case, equilibrium policies may be non-quantized and even linear. In the noisy setup, a Gaussian source is to be transmitted over an additive Gaussian channel. The goals of the encoder and the decoder are misaligned by a bias term and encoder's cost 
also includes a power term scaled by a multiplier. Conditions for the existence of affine equilibrium policies as well as general informative equilibria are presented for both the scalar and multi-dimensional setups. We compare the results with socially optimal costs and information theoretic lower bounds, and discuss the effects of the bias term. Due to space constraints, the sketches of a number of results are presented. Details are included in [20].

\section{FORMAL DESCRIPTION}

Let there be two decision makers (DMs): An encoder (DM 1) and a decoder (DM 2). DM 1 wishes to encode the $\mathbb{M}$-valued random variable $M$ to DM 2 . Let $X$ denote the $\mathbb{X}$ valued random variable which is transmitted to DM 2. DM 2, upon receiving $X$, generates its optimal decision $U$ which we also take to be $\mathbb{M}$-valued. We allow for randomized decisions, therefore, we let the policy space of DM 1 be the set of all stochastic kernels from $M$ to $X$. Recall that $P$ is a stochastic kernel from $\mathbb{M}$ to $\mathbb{X}$ if $P(\cdot \mid X=x)$ is a probability measure on $\mathcal{B}(\mathbb{X})$ for every $x \in \mathbb{M}$ and for every Borel $A, P(A \mid X=x)$ is a measurable function of $x$. Let $\Gamma^{e}$ denote the set of all such policies. We let the policy space of DM 2 be the set of all stochastic kernels from $X$ to $U$. Let $\Gamma^{d}$ denote the set of all such stochastic kernels.

Given $\gamma^{e} \in \Gamma^{e}$ and $\gamma^{d} \in \Gamma^{d}$, the goal in the classical communications theory is to minimize the expectation

$$
J\left(\gamma^{e}, \gamma^{d}\right)=\int c(m, u) \gamma^{e}(d x \mid m) \gamma^{d}(d u \mid x) P(d m),
$$

where $c$ is some cost function. Typical applications have $c(m, u)=|m-u|^{2}$. It is well-known that for such problems the extreme solutions are the most informative ones: Optimal encoders and decoders are deterministic with as high information rate as possible.

Recall that a collection of decision makers who have an agreement on the probabilistic description of a system and a cost function to be minimized, but who may have different on-line information is said to be a team (see, e.g. [21]). Hence, the classical communications setup may be viewed as a team of an encoder and a decoder.

In many applications (in networked systems, recommendation systems, and applications in economics) the objectives of the encoder and the decoder may not be aligned. For example, DM 1 may aim to minimize

$$
J^{e}\left(\gamma^{e}, \gamma^{d}\right)=\int c^{e}(m, u) \gamma^{e}(d x \mid m) \gamma^{d}(d u \mid x) P(d m),
$$

whereas DM 2 may aim to minimize

$$
J^{d}\left(\gamma^{e}, \gamma^{d}\right)=\int c^{d}(m, u) \gamma^{e}(d x \mid m) \gamma^{d}(d u \mid x) P(d m) \text {. }
$$

Such a problem is known in the economics literature as cheap talk. A more general formulation would be the case when the transmitted signal is also an explicit part of the cost function $c^{e}$ or $c^{d}$; in that case, the setup is called a signaling game. We will consider a noisy communication setup, where the problem may be viewed as a signaling game, rather than cheap talk, later in this study.
Since the goals are not aligned, such a problem is studied under the tools and concepts provided by game theory. A pair of policies $\gamma^{*, e}, \gamma^{*, d}$ is said to be a Nash equilibrium if

$$
\begin{array}{ll}
J^{e}\left(\gamma^{*, e}, \gamma^{*, d}\right) \leq J^{e}\left(\gamma^{e}, \gamma^{*, d}\right) & \forall \gamma^{e} \in \Gamma^{e} \\
J^{d}\left(\gamma^{*, e}, \gamma^{*, d}\right) \leq J^{d}\left(\gamma^{*, e}, \gamma^{d}\right) & \forall \gamma^{d} \in \Gamma^{d}
\end{array}
$$

We note that when $c^{e}=c^{d}$ the setup is a traditional communication theoretic setup. If $c^{e}=-c^{d}$, that is, if the setup is a zero-sum game, then an equilibrium is achieved when $\gamma^{e}$ is non-informative (e.g., a kernel with actions statistically independent of the source) and $\gamma^{d}$ uses only the prior information (since the received information is non-informative). We call such an equilibrium a non-informative equilibrium. The following can be shown:

Proposition 2.1: A non-informative equilibrium always exists for the cheap talk game.

Crawford and Sobel [6] have made foundational contributions to the study of cheap talk with misaligned objectives where the cost functions $c^{e}$ and $c^{d}$ satisfy certain monotonicity and differentiability properties but there is a bias term in the cost functions for a uniform source. Their result is that the number of bins in an equilibrium is upper bounded by a function which is negatively correlated to the bias.

\section{A. Equilibrium, price of anarchy, and the issue of commit- ment}

In this paper, we take the cost functions as

$$
c^{e}(m, u)=(m-u-b)^{2}, \quad c^{d}(m, u)=(m-u)^{2} .
$$

where $b$ denotes the bias term. The motivation for such functions stems from the fields of information theory, communication theory and LQG control. Recall that for the $b=0$ case, the cost functions simply reduce to a minimum mean-square estimation (MMSE) problem.

The formulation considered in this study focuses on setups where there is not necessarily a commitment: If the encoder and the decoder take part in a repeated game and are committed to their announced policies, they should play according to a policy that minimizes the total cost: $J^{e}\left(\gamma^{e}, \gamma^{d}\right)+$ $J^{d}\left(\gamma^{e}, \gamma^{d}\right)$; which results in $2 \inf _{\gamma^{e}, \gamma^{d}} E\left[\left(m-u-\frac{b}{2}\right)^{2}\right]+\frac{b^{2}}{2}$. However, a lack of commitment may lead one of the players to deviate from their strategy and pick another function, hence the need for a game theoretic solution arises.

Among the equilibria, one particular interest is on the most informative ones: The (socially optimal) equilibrium with the smallest $J^{e}\left(\gamma^{*, e}, \gamma^{*, d}\right)+J^{d}\left(\gamma^{*, e}, \gamma^{*, d}\right)$. As is common in game theoretic problems, a socially optimal solution which minimizes the sum $J^{e}\left(\gamma^{e}, \gamma^{d}\right)+J^{d}\left(\gamma^{e}, \gamma^{d}\right)$ is typically not achieved in any equilibrium, as we will observe later in the manuscript.

\section{B. Structural results on equilibrium policies}

Let the cost functions defined as $c^{e}(m, u)=(m-u-b)^{2}$ and $c^{d}(m, u)=(m-u)^{2}$ where $b$ is bias term. Some existence and deterministic properties of the equilibrium policies 
of the encoder and the decoder are stated in [19] and [21, Chp.4].

Theorem 2.1: [19] (i) For any $\gamma^{e}$, there exists an optimal $\gamma^{d}$, which is deterministic. (ii) For any $\gamma^{d}$, any randomized encoding policy can be replaced with a deterministic $\gamma^{e}$ without any loss to DM 1. (iii) Suppose $\gamma^{e}$ is an $M$ cell quantizer, then there exists an optimal $\gamma^{d}$, which is the conditional expectation of the respective bin.

The following builds on [6, Lem.1], which considers uniform scalar sources. We note that the analysis here applies to arbitrary scalar valued random variables.

Theorem 2.2: An equilibrium policy has to be quantized (or is equivalent to a quantized policy) for the encoder cost function $c^{e}(m, u)=(m-u-b)^{2}$ and the decoder cost function $c^{d}(m, u)=(m-u)^{2}$ where $m$ is any scalar random source and $b \neq 0$.

Proof: Let there be an equilibrium in the game (with possibly uncountably infinitely many bins, countably many bins or finitely many bins). Let two bins be $\mathcal{B}^{\alpha}$ and $\mathcal{B}^{\beta}$. Also let $m^{\alpha}$ indicate any point in $\mathcal{B}^{\alpha}$; i.e., $m^{\alpha} \in \mathcal{B}^{\alpha}$. Similarly, let $m^{\beta}$ indicate any point in $\mathcal{B}^{\beta}$; i.e., $m^{\beta} \in \mathcal{B}^{\beta}$. The decoder chooses the action $u^{\alpha}=E\left[m \mid m \in \mathcal{B}^{\alpha}\right]$ when the encoder sends $m^{\alpha} \in \mathcal{B}^{\alpha}$ and action $u^{\beta}=E\left[m \mid m \in \mathcal{B}^{\beta}\right]$ when the encoder sends $m^{\beta} \in \mathcal{B}^{\beta}$ in order to minimize its total cost. Without loss of generality, we can assume that $m^{\alpha}<m^{\beta}$, hence $u^{\alpha}<u^{\beta}$. Because of the equilibrium definitions from the view of the encoder; $\left(m^{\alpha}-u^{\alpha}-b\right)^{2}<\left(m^{\alpha}-u^{\beta}-\right.$ $b)^{2}$ and $\left(m^{\beta}-u^{\beta}-b\right)^{2}<\left(m^{\beta}-u^{\alpha}-b\right)^{2}$. Hence, $\exists$ $\bar{m}$ that satisfies $\left(\bar{m}-u^{\alpha}-b\right)^{2}=\left(\bar{m}-u^{\beta}-b\right)^{2}$ which reduces to $\bar{m}=\frac{u^{\alpha}+u^{\beta}}{2}+b \Rightarrow\left(\bar{m}-u^{\alpha}\right)=\left(u^{\beta}-\bar{m}\right)+2 b$. Recall that $m^{\alpha}<\frac{2}{m}<m^{\beta}$ due to the continuity of the cost functions and this implies $u^{\alpha}<\bar{m}<u^{\beta}$. It then follows that $u^{\beta}-u^{\alpha}=\left(u^{\beta}-\bar{m}\right)+\left(\bar{m}-u^{\alpha}\right)>2|b|$. Thus, there must be at least $2|b|$ distance between the equilibrium points (decoder's actions, centroids of the bins), which guarantees that the equilibrium policy must be discrete.

Recall again that for the case when the source is uniform, Crawford and Sobel established the discrete nature of the equilibrium policies. For the case when the source is exponential, [19] established the discrete-nature, and obtained the equilibrium bins with finite upper bounds on the number of bins in any equilibrium. For the Gaussian source case, obtaining an analytical solution appears to be difficult due to the complicated integrations involved, but the quantized nature can easily be established: The Gaussian case is important because if the costs are aligned, optimal encoder and decoder policies are always linear in a team-theoretic setup: When $b$ is non-zero, this may not be true. We will revisit this topic later.

\section{Multi-Dimensional Cheap Talk}

Let the source be uniform on $[0,1] \times[0,1]$ and the cost function of the encoder be defined by $c^{e}(\vec{m}, \vec{u})=\| \vec{m}-\vec{u}-$ $\vec{b} \|^{2}$ where the length of the vectors is defined in $L_{2}$ norm.

Theorem 3.1: An equilibrium policy, unlike the scalar case, can be non-discrete and in fact linear.

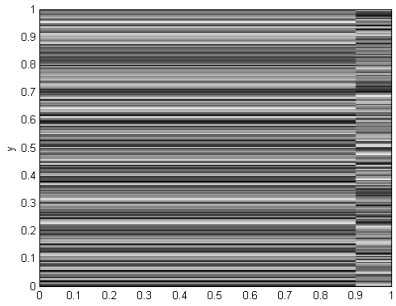

(a) There are 2 quantization levels on the $x$-dimension and 200 quantization levels on the $y$-dimension. The number of quantization levels on the $y$-dimension can be arbitrarily chosen (since $\vec{b}$ is orthogonal to that dimension). As the number of levels goes to infinity, this construction converges to the structure of a linear equilibrium.

Fig. 1: Sample equilibria in 2D

Proof: It suffices to provide an example. Consider $\vec{b}=[0.20]$. Then, as a limit case of the equilibrium in Fig. 1a, the following encoder and decoder policies form an equilibrium:

$$
\begin{gathered}
\gamma^{e}\left(m_{1}, m_{2}\right)=\left(x_{1}, x_{2}\right)= \begin{cases}\left(C^{1}, m_{2}\right) & \text { if } m_{1} \in[0.0,0.9] \\
\left(C^{2}, m_{2}\right) & \text { if } m_{1} \in(0.9,1.0]\end{cases} \\
\gamma^{d}\left(x_{1}, x_{2}\right)=\left(u_{1}, u_{2}\right)= \begin{cases}\left(0.45, m_{2}\right) & \text { if } x_{1}=C^{1} \\
\left(0.95, m_{2}\right) & \text { if } x_{1}=C^{2}\end{cases}
\end{gathered}
$$

where $C^{1}$ and $C^{2}$ are any two constants. Here, the scalar setup is applied on the $x$-dimension with two quantization bins (recall that $u_{1}=E\left[m_{1} \mid x_{1}=C^{1}\right]$ or $u_{1}=E\left[m_{1} \mid x_{1}=\right.$ $\left.C^{2}\right]$ ), and a fully-informative equilibrium exists on the $y$ dimension since there is no bias on that dimension. It is observed that the encoder and decoder have linear policies due to the unbiased property of the $y$-dimension.

Besides linear equilibria, there may be multiple (hence, non-unique) quantized equilibria with finite regions in the multi-dimensional case as illustrated in Fig. $1 \mathrm{~b}$.

Remark 3.1: If $\vec{b}$ is orthogonal to the basis vectors or satisfies certain symmetry conditions, then linear equilibria exist; namely, the presence of linear equilibria is a rare occurrence. This argument applies also to an $n$-dimensional setup for any $n \in \mathbb{N}$.

\section{Quadratic Signaling Game: Scalar Case}

The noisy game setup is similar to the noiseless case except that there exists an additive Gaussian noise channel between the encoder and decoder, as shown in Fig. 2, and the encoder has a soft power constraint.

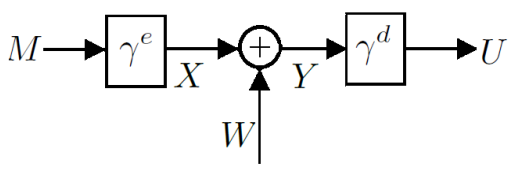

Fig. 2: General system model. 
The encoder (DM 1) encodes a zero-mean Gaussian random variable $M$ and sends the real-valued random variable $X$. During the transmission, the zero mean Gaussian noise with a variance of $\sigma^{2}$ is added to $X$; hence, the decoder (DM 2) receives $Y=X+W$. The policy space of DM 1, $\Gamma^{e}$, is similarly defined as the policy space in the noiseless case: the set of stochastic kernels from $\mathbb{R}$ to $\mathbb{R}$ (this can be viewed as the measurable subset of the space of all product measures involving $M, X$ with a fixed input marginal, under the weak topology). The policy space of DM $2, \Gamma^{d}$, is the set of stochastic kernels from $Y$ to $U$. The cost functions of the encoder and the decoder are also slightly modified as follows: DM 1 aims to minimize

$$
J^{e}(\bar{\gamma})=\int c^{e}(m, u) \gamma^{e}(d x \mid m) \gamma^{d}(d u \mid y) P(d y \mid x) P(d m),
$$

whereas DM 2 aims to minimize

$$
J^{d}(\bar{\gamma})=\int c^{d}(m, u) \gamma^{e}(d x \mid m) \gamma^{d}(d u \mid y) P(d y \mid x) P(d m),
$$

where $\bar{\gamma}=\left(\gamma^{e}, \gamma^{d}\right)$ and $P(d y \mid x)=P(W \in d y-x)$ with $W \sim \mathcal{N}\left(0, \sigma^{2}\right)$. The cost functions are modified as follows:

$c^{e}(m, x, u)=(m-u-b)^{2}+\lambda x^{2}, c^{d}(m, u)=(m-u)^{2}$

Note that a power constraint with an associated multiplier is appended to the cost function of the encoder, which corresponds to power limitation for transmitters in practice. If $\lambda=0$, this corresponds to the setup with no power constraint at the encoder.

\section{A. A Supporting Result}

Suppose that there is an equilibrium with an arbitrary policy leading to finite (at least two), countably infinite or uncountably infinite equilibrium bins. Let two of these bins be $\mathcal{B}^{\alpha}$ and $\mathcal{B}^{\beta}$. Also let $m^{\alpha}$ indicate any point in $\mathcal{B}^{\alpha}$; i.e., $m^{\alpha} \in \mathcal{B}^{\alpha}$; and the encoder encodes $m^{\alpha}$ to $x^{\alpha}$ and sends to the decoder. Similarly, let $m^{\beta}$ indicate any point in $\mathcal{B}^{\beta}$; i.e., $m^{\beta} \in \mathcal{B}^{\beta}$; and the encoder encodes $m^{\beta}$ to $x^{\beta}$ and sends to the decoder. Without any loss of generality, we can assume that $m^{\alpha}<m^{\beta}$. The decoder chooses the action $u=E[m \mid y]$ (MMSE rule). Let $F(m, x)$ be the encoder cost when message $m$ is encoded as $x$; i.e.,

$$
\begin{aligned}
F(m, x)=\int_{y} p\left(\gamma^{d}(y)=u \mid \gamma^{e}(m)=\right. & x)\left((m-u-b)^{2}\right. \\
& \left.+\lambda x^{2}\right) \mathrm{d} y
\end{aligned}
$$

Then the equilibrium definitions from the view of the encoder requires $F\left(m^{\alpha}, x^{\alpha}\right) \leq F\left(m^{\alpha}, x^{\beta}\right)$ and $F\left(m^{\beta}, x^{\beta}\right) \leq$ $F\left(m^{\beta}, x^{\alpha}\right)$. Now let $G(m)=F\left(m, x^{\alpha}\right)-F\left(m, x^{\beta}\right)$. Since $G(m)$ is a continuous function of $m$ on the interval $\left[m^{\alpha}, m^{\beta}\right]$, $\exists \bar{m} \in\left[m^{\alpha}, m^{\beta}\right]$ such that $G(\bar{m})=0$ by the Mean Value Theorem, which implies $F\left(\bar{m}, x^{\alpha}\right)=F\left(\bar{m}, x^{\beta}\right)$. Then by writing the integrals as expectations, the following is obtained:

$$
\bar{m}=\frac{E\left[\left(\gamma^{d}(y)\right)^{2} \mid x^{\beta}\right]-E\left[\left(\gamma^{d}(y)\right)^{2} \mid x^{\alpha}\right]+\lambda\left(\left(x^{\beta}\right)^{2}-\left(x^{\alpha}\right)^{2}\right)}{2\left(E\left[\gamma^{d}(y) \mid x^{\beta}\right]-E\left[\gamma^{d}(y) \mid x^{\alpha}\right]\right)}+b
$$

Recall that the arguments in Theorem 2.2 cannot be applied here because of the presence of noise. However, when there is noise in a communication channel, the relation between $E[u \mid x], E\left[u^{2} \mid x\right]$ and $\bar{m}$ can be constructed as in (5).

\section{B. Existence and Uniqueness of Informative Equilibria and Affine Equilibria}

We first note that Proposition 2.1 is valid also in the noisy formulation; i.e. a non-informative equilibrium is an equilibrium for the noisy signaling game, since the appended power constraint is always positive. The following holds:

Theorem 4.1: 1) Let $0<\lambda<\frac{E\left[m^{2}\right]}{E\left[w^{2}\right]}$. For any $b \in \mathbb{R}$, there exists a unique informative affine equilibrium.

2) If $\lambda \geq \frac{E\left[m^{2}\right]}{E\left[w^{2}\right]}$, there does not exist an informative (affine or non-linear) equilibrium. The only equilibrium is the non-informative one.

3) If $\lambda=0$, there exists no informative equilibrium with affine policies.

Sketch of Proof:

1) If the encoder is linear (affine), the decoder, as an MMSE decoder for a Gaussian source over a Gaussian channel, is linear (affine); this follows from the property of the conditional expectation for jointly Gaussian random variables; i.e., with the given affine encoding policy $x=\gamma^{e}(m)=A m+C$, the optimal decoder policy would be

$$
\gamma^{d}(y)=K y+L=\frac{A E\left[m^{2}\right]}{A^{2} E\left[m^{2}\right]+E\left[w^{2}\right]}(y-C)
$$

Further, if the decoder is affine so that $u=\gamma^{d}(y)=$ $K y+L$, it can be shown that the optimal encoder is also affine:

$$
\gamma^{*, e}(m)=A m+C=\frac{(m-L-b)}{K+\lambda / K}
$$

By combining (6) and (7), we obtain $\left(K^{2}+\lambda\right)^{2} E\left[w^{2}\right]=$ $\lambda E\left[\mathrm{~m}^{2}\right]$ by assuming $A \neq 0$; which implies $K^{2}=$ $\sqrt{\frac{\lambda E\left[m^{2}\right]}{E\left[w^{2}\right]}}-\lambda$. If we combine the equations above by using $A$ and define the resulting mapping as $T(A)$, we obtain

$$
A=T(A)=\frac{\frac{A}{A^{2}+E\left[w^{2}\right] / E\left[m^{2}\right]}}{\left(\frac{A}{A^{2}+E\left[w^{2}\right] / E\left[m^{2}\right]}\right)^{2}+\lambda} .
$$

can be viewed as a continuous function mapping the compact convex set $\left[0, \max \left(E\left[m^{2}\right] / E\left[w^{2}\right], 1\right) / \lambda\right]$ to itself. Therefore, by Brouwer's fixed point theorem [22], there exists $A=T(A)$. Indeed, we can find nonzero $A, K, C$ and $L$ for every $0<\lambda<\frac{E\left[m^{2}\right]}{E\left[w^{2}\right]}$ from the equilibrium equations in (6) and (7). For the uniqueness of an informative fixed point, suppose that there are two different nonzero fixed points $A_{1}$ and $A_{2}$ such that $A_{1}=T\left(A_{1}\right)$ and $A_{2}=T\left(A_{2}\right)$. Then $A_{1} / T\left(A_{1}\right)=A_{2} / T\left(A_{2}\right)$ implies $\left|A_{1}\right|=\left|A_{2}\right|$, and since the mapping is defined from $\left[0, \max \left(E\left[m^{2}\right] /\right.\right.$ $\left.\left.E\left[w^{2}\right], 1\right) / \lambda\right]$ to itself, the nonzero fixed point is unique. 
Then the encoder may choose the nonzero fixed point for the informative equilibrium if it results in a lower cost than the non-informative equilibrium (due to the cost of communication, an informative equilibrium is not always beneficial to the encoder compared to the non-informative one).

2) Let $\lambda \geq E\left[m^{2}\right] / E\left[w^{2}\right]$ and suppose that we are in an equilibrium. Then, the encoder cost $J^{e}=E[(m-$ $\left.u-b)^{2}+\lambda x^{2}\right]$ reduces to $J^{e}=E\left[(m-u)^{2}\right]+$ $\lambda E\left[x^{2}\right]+b^{2}$, and since the decoder in an equilibrium always chooses $u=E[m \mid y]$, through $P=E\left[x^{2}\right]$, the following analysis leads to a lower bound on the encoder cost:

$$
\begin{aligned}
J^{e} & =b^{2}+\lambda E\left[x^{2}\right]+E\left[(m-u)^{2}\right] \\
& \geq b^{2}+\lambda P+E\left[m^{2}\right] \mathrm{e}^{-2 \sup I(X ; Y)} \\
& =b^{2}+\lambda P+E\left[m^{2}\right] \mathrm{e}^{-2 \frac{1}{2} \log \left(1+\frac{P}{E\left[w^{2}\right]}\right)} \\
& =b^{2}+\lambda P+\frac{E\left[m^{2}\right]}{1+P / E\left[w^{2}\right]} .
\end{aligned}
$$

Here, (9) follows from a rate-distortion theoretic bound through the data-processing inequality (see for example p. 96 of [21]). However, it follows that when $\lambda \geq E\left[m^{2}\right] / E\left[w^{2}\right],(10)$ is minimized at $P=0$; that is, the encoder does not signal any output. Hence, the encoder engages in an non-informative equilibrium and the minimum cost becomes $E\left[m^{2}\right]+b^{2}$ at this non-informative equilibrium.

3) It is proved that an optimal encoder is affine such that $x=\gamma^{e}(m)=A m+C$ when the decoder is affine, that is, $u=\gamma^{d}(y)=K y+L$. Then, by inserting $\lambda=0$ to (5), $\bar{m}$ is obtained as

$$
\bar{m}=K A \frac{\left(m^{\alpha}+m^{\beta}\right)}{2}+K C+L+b .
$$

The above holds for all $m^{\alpha}$ and $m^{\beta}$ with $m^{\alpha} \leq \bar{m} \leq$ $m^{\beta}$. Thus, if the distance between $m^{\alpha}$ and $m^{\beta}$ is made arbitrarily small, then it must be that $K A=1$ and $K C+L+b=0$. By combining these and (6), it follows that a real solution does not exist for any given affine coding parameter.

Remark 4.1: If either $\lambda$ or $E\left[w^{2}\right]$ is 0 , an informative affine equilibrium exists only if $\lambda, E\left[w^{2}\right]$ and $b$ are all 0 .

\section{Price of Anarchy and Comparison with Socially Optimal Cost}

In this part, it will be shown that the game theoretic cost is higher than the socially optimal cost as expected, and the information theoretic lower bounds on the costs and their achievability will be discussed.

Theorem 4.2: There always exists a price of anarchy in the game setup in the sense that the sum of the costs under any equilibria is always larger than the socially optimal cost.

In the following, we discuss information theoretic lower bounds on the performance of equilibria and socially optimal strategies. The theorem below is based on the approach outlined in (10).

Theorem 4.3: 1) For the game setup, if $\lambda \geq \frac{E\left[m^{2}\right]}{E\left[w^{2}\right]}$ (i.e., non-informative equilibria), the information theoretic lower bounds on the costs are achievable.

2) For the game setup, if $\lambda<\frac{E\left[m^{2}\right]}{E\left[w^{2}\right]}$ and $b=0$, then the information theoretic lower bounds on the costs are achievable by linear policies.

3) For the game setup, if $\lambda<\frac{E\left[m^{2}\right]}{E\left[w^{2}\right]}$ and $b \neq 0$, the information theoretic lower bounds on the costs are not achievable by affine policies.

4) For the team setup, the information theoretic lower bounds on the costs are always (both in the informative and non-informative equilibria) achievable by affine policies.

\section{Quadratic Signaling Game: Multi-Dimensional Gaussian Noisy CAsE}

The scalar setup considered in Section IV can be extended to the multi-dimensional Gaussian noisy signaling game problem setup as follows. The encoder (DM 1) encodes an $n$-dimensional zero-mean Gaussian random variable $\vec{M}$ and sends the real-valued $n$-dimensional random variable $\vec{X}$. During the transmission, the $n$-dimensional zeromean Gaussian noise with the covariance matrix $\Sigma_{\vec{W}}$ is added to $\vec{X}$ and the decoder (DM 2) receives $\vec{Y}=\vec{X}+\vec{W}$. The policy space of DM $1, \Gamma^{e}$, is the set of stochastic kernels from $\mathbb{R}^{n}$ to $\mathbb{R}^{n}$. The policy space of DM $2, \Gamma^{d}$, is the set of stochastic kernels from $\vec{Y}$ to $\vec{U}$. The cost functions of the encoder and the decoder are as follows: DM 1 aims to minimize

$$
J^{e}(\bar{\gamma})=\int c^{e}(\vec{m}, \vec{u}) \gamma^{e}(d \vec{x} \mid \vec{m}) \gamma^{d}(d \vec{u} \mid \vec{y}) P(d \vec{y} \mid \vec{x}) P(d \vec{m}),
$$

whereas DM 2 aims to minimize

$$
J^{d}(\bar{\gamma})=\int c^{d}(\vec{m}, \vec{u}) \gamma^{e}(d \vec{x} \mid \vec{m}) \gamma^{d}(d \vec{u} \mid \vec{y}) P(d \vec{y} \mid \vec{x}) P(d \vec{m}),
$$

where $\bar{\gamma}=\left(\gamma^{e}, \gamma^{d}\right)$ and $P(d \vec{y} \mid \vec{x})=P(\vec{W} \in d \vec{y}-\vec{x})$ with $\vec{W} \sim \mathcal{N}\left(0, \Sigma_{\vec{W}}\right)$. The cost functions are

$c^{e}(\vec{m}, \vec{x}, \vec{u})=\|\vec{m}-\vec{u}-\vec{b}\|^{2}+\lambda\|\vec{x}\|^{2}, c^{d}(\vec{m}, \vec{u})=\|\vec{m}-\vec{u}\|^{2}$.

Note that we have appended a power constraint and an associated multiplier. If $\lambda=0$, this corresponds to the setup with no power constraint at the encoder.

\section{A. Affine Equilibria}

Theorem 5.1: 1) If the encoder is linear (affine), then an optimal decoder policy is linear (affine).

2) If the decoder is linear (affine), then an optimal encoder policy is an affine policy.

3) For $\lambda>0$, there exists an affine equilibrium in the multi dimensional Gaussian noisy signaling game.

Sketch of Proof: 
1) Let the affine encoding policy be $\gamma^{e}(\vec{m})=A \vec{m}+\vec{C}$. Then the optimal decoder, as an MMSE decoder for a Gaussian source over a Gaussian channel, is affine:

$$
\begin{array}{r}
\gamma^{*, d}(\vec{y})=K \vec{y}+\vec{L}=\Sigma_{\vec{M}} A^{T}\left(A \Sigma_{\vec{M}} A^{T}+\Sigma_{\vec{W}}\right)^{-1} \\
(\vec{y}-\vec{C}) \quad(11)
\end{array}
$$

2) Let the affine decoding policy be $\vec{u}=\gamma^{d}(\vec{y})=K \vec{y}+$ $\vec{L}$. It can be shown that the optimal encoder is affine:

$$
\begin{array}{r}
\gamma^{*, e}(\vec{m})=A \vec{m}+\vec{C}=\left(K^{T} K+\lambda I\right)^{-1} K^{T} \\
(\vec{m}-\vec{L}-\vec{b})
\end{array}
$$

3) From (11) and (12), we have $A=\left(K^{T} K+\lambda I\right)^{-1} K^{T}$ and $K=\Sigma_{\vec{M}} A^{T}\left(A \Sigma_{\vec{M}} A^{T}+\Sigma_{\vec{W}}\right)^{-1}$. By combining these, we obtain the following:

$$
A=T(A)=\left(F F^{T}+\lambda I\right)^{-1} F
$$

where $F=\left(A \Sigma_{\vec{M}} A^{T}+\Sigma_{\vec{W}}\right)^{-1} A \Sigma_{\vec{M}}$. (13) implies a mapping and this mapping is denoted by $T(A)$. Recall that the one-dimensional equivalent of (13) is (8); hence, the results are consistent. Since $F F^{T}$ is a real and symmetric matrix, then it is diagonalizable and can be written as $F F^{T}=Q \Upsilon Q^{-1}$. Now consider $\|T(A)\|_{F}$ :

$$
\|T(A)\|_{F}=\operatorname{tr}\left(A A^{T}\right)=\sum_{i=1}^{n} \frac{v_{i}}{\left(v_{i}+\lambda\right)^{2}}
$$

where $v_{i}, i=1, \ldots, n$ are the eigenvalues of $F F^{T}$ and since $F F^{T}$ is positive semi-definite, all these eigenvalues are nonnegative. Assuming the nonzero power constraint $(\lambda>0), v_{i} /\left(v_{i}+\lambda\right)^{2}<\max \left(1,1 / \lambda^{2}\right)$ always holds. Then, by (14), we have $\|T(A)\|_{F}<$ $n \max \left(1,1 / \lambda^{2}\right)$, which implies that $T(A)$ can be viewed as a continuous function mapping the compact convex set $\|A\|_{F} \in\left[0, n \max \left(1,1 / \lambda^{2}\right)\right]$ to itself. Therefore, by Brouwer's fixed point theorem [22], there exists $A=T(A)$.

We note, however, that there always exist a non-informative equilibrium (see Proposition 2.1, which also applies to the signaling game discussed in this section). However, there exist games with informative affine equilibria as we state in the following (see Theorem 5.2).

Proposition 5.1: If either $\lambda$ or $\Sigma_{\vec{W}}$ is zero, an informative affine equilibrium exists only if $\lambda, \Sigma_{\vec{W}}$ and $\vec{b}$ are all zero.

Remark 5.1: In the multi-dimensional case, fixed points may not be unique.

Theorem 5.2: Let source $\vec{M}$ be an $n$-dimensional Gaussian zero-mean random variable with covariance matrix $\Sigma_{\vec{M}}$ $=\operatorname{diag}\left\{\sigma_{m_{1}}^{2}, \cdots, \sigma_{m_{n}}^{2}\right\}$ where diag indicates a diagonal matrix, and noise $\vec{W}$ be a zero-mean $n$-dimensional Gaussian random variable with covariance matrix $\Sigma_{\vec{W}}=$ diag $\left\{\sigma_{w_{1}}^{2}, \cdots, \sigma_{w_{n}}^{2}\right\}$. Then an informative affine equilibrium exists if $\lambda<\max \left\{\frac{\sigma_{m_{1}}^{2}}{\sigma_{w_{1}}^{2}}, \cdots, \frac{\sigma_{m_{n}}^{2}}{\sigma_{w_{n}}^{2}}\right\}$.

\section{CONCLUding Remarks}

It has been shown that the equilibrium policies may be non-discrete and even linear for a multi-dimensional cheap talk problem which is different from the scalar case. Furthermore, for a quadratic Gaussian signaling game problem, conditions for the existence of affine equilibrium policies as well as general informative equilibria are presented for both the scalar and multi-dimensional setups. Our findings provide further conditions on when affine policies may be optimal in decentralized multi-criteria control problems and lead to conditions for the presence of active information transmission in strategic environments.

\section{REFERENCES}

[1] T. Başar and G. Olsder, Dynamic Noncooperative Game Theory. Philadelphia, PA: SIAM Classics in Applied Mathematics, 1999.

[2] I. Shames, A. Teixeira, H. Sandberg, and K. Johansson, "Agents misbehaving in a network: a vice or a virtue?" IEEE Network, vol. 26, no. 3, pp. 35-40, May 2012.

[3] Y. Mo, T.-H. Kim, K. Brancik, D. Dickinson, H. Lee, A. Perrig, and B. Sinopoli, "Cyber physical security of a smart grid infrastructure," Proceedings of the IEEE, vol. 100, no. 1, pp. 195-209, Jan. 2012.

[4] T. Alpcan and T. Başar, Network Security: A Decision and GameTheoretic Approach, 1st ed. New York, NY, USA: Cambridge University Press, 2010.

[5] J. Miklós-Thal and H. Schumacher, "The value of recommendations," Games and Economic Behavior, vol. 79, pp. 132-147, 2013.

[6] V. P. Crawford and J. Sobel, "Strategic information transmission," Econometrica, vol. 50, pp. 1431-1451, 1982.

[7] V. Krishna and J. Morgan, "A model of expertise," The Quarterly Journal of Economics, vol. 116, no. 2, pp. 747-775, 2001.

[8] S. Miura, "Strategic communication games: Theory and applications," $\mathrm{Ph} . \mathrm{D}$. dissertation, Washington University in St. Louis, 2012.

[9] M. Battaglini, "Multiple referrals and multidimensional cheap talk," Econometrica, vol. 70, no. 4, pp. 1379-1401, 2002.

[10] G. Levy and R. Razin, "On the limits of communication in multidimensional cheap talk: A comment," Econometrica, vol. 75, no. 3, pp. pp. 885-893, 2007.

[11] A. Chakraborty and R. Harbaugh, "Comparative cheap talk," Journal of Economic Theory, vol. 132, no. 1, pp. 70 - 94, 2007.

[12] A. Ambrus and S. Takahashi, "Multi-sender cheap talk with restricted state space," Theoretical Economics, vol. 3, no. 1, pp. 1-27, Mar. 2008

[13] A. Blume, O. J. Board, and K. Kawamura, "Noisy talk," Theoretical Economics, vol. 2, no. 4, Dec. 2007.

[14] Y. Chen, "Communication with two-sided asymmetric information," 2009, working paper.

[15] I. M. de Barreda, "Cheap talk with two-sided private information," 2010, working paper.

[16] E. K. Lai, "Expert advice for amateurs," Journal of Economic Behavior Organization, vol. 103, pp. 1 - 16, 2014.

[17] M. Golosov, V. Skreta, A. Tsyvinski, and A. Wilson, "Dynamic strategic information transmission," Journal of Economic Theory, vol. 151, pp. 304 - 341, 2014.

[18] F. Farokhi, A. M. H. Teixeira, and C. Langbort, "Estimation with strategic sensors," CoRR, vol. abs/1402.4031, 2014. [Online]. Available: http://arxiv.org/abs/1402.4031

[19] S. Fabricius, P. Furrer, S. Kerner, T. Linder, and S. Yüksel, "Game theory and information, Queen's University, MTHE 493 Technical Report," Apr. 2014.

[20] S. Sarıtaş, S. Gezici, and S. Yüksel, "Quadratic multi-dimensional signaling games and affine equilibria," arXiv.

[21] S. Yüksel and T. Başar, Stochastic Networked Control Systems: Stabilization and Optimization under Information Constraints. Boston, MA: Birkhäuser, 2013.

[22] C. D. Aliprantis and K. C. Border, Infinite Dimensional Analysis: A Hitchhikers Guide, 3rd ed. Berlin: Springer-Verlag, 2006. 